\title{
Can Left ventricular outflow tract aortic velocity time integral guide fluid resuscitation in septic patients? - A case report
}

\author{
Livio Colombo, ${ }^{1}$ Francesco Panizzardi, ${ }^{1}$ Irene Rusconi, ${ }^{1}$ Anna Roncoroni, ${ }^{1}$ Marta Bergamaschi, ${ }^{2}$ \\ Alfredo Macheda ${ }^{1}$
}

\author{
${ }^{1}$ Pronto Soccorso - DEA Ospedale SantiPaoloCarlo, Presidio San Paolo, Milan; ${ }^{2}$ Pronto Soccorso - \\ Medicina d'Urgenza Ospedale Niguarda, Milan, Italy
}

\begin{abstract}
Hemodynamic monitoring of unstable patients is an everyday issue for Emergency Physicians (EP). Considering the difficulty, in Emergency Department (ED) settings, to assess invasively Stroke Volume (SV), Cardiac Output (CO) and Peripheral Vascular Resistance (PVR), EP should be familiar with non-invasive, easy and reproducible methods that can estimate these parameters. The use of Left Ventricular Outflow Tract aortic Velocity Time Integral (LVOT-VTI) with echocardiography, as estimate of SV, integrated with inferior vena cava collapse index and clinical examination could give the opportunity to non-invasively understand at which point of an ideal cardiac output/central venous pressure relation (according to the Frank Starling law) the patient is situated. In this case report we describe a septic patient accessing the ED with both respiratory and cardiac failure, and we show that the use of aortic LVOT-VTI is an easy and reproducible approach to understand cardiac hemodynamic in scenarios involving multiple pathologic mechanisms.
\end{abstract}

\section{Case Report}

We describe the case of an 81 years old woman coming in the ED hypotensive, fever over $38^{\circ}$ in the past 4 days with cough, yellowish sputum, progressive worsening shortness of breath and

\footnotetext{
Correspondence: Livio Colombo, Via Eugenio Pellini 4, 20125, Milan, Italy

E-mail: liviocolombo@me.com

Tel.: +39.3497853013

Key words: Aortic velocity time integral; unstable patients; hemodynamic monitoring.

Ethics approval and consent to participate: The manuscript does not contain any elements that would allow the recognition of the patient. Conflict of interest: the authors declare no potential conflict of interests.

Received for publication: 12 January 2020.

Accepted for publication: 6 June 2020.

This work is licensed under a Creative Commons Attribution 4.0 License (by-nc 4.0).

${ }^{\circ}$ Copyright: the Author(s), 2020

Licensee PAGEPress, Italy

Emergency Care Journal 2020; 16:8817

doi:10.4081/ecj.2020.8817
}

increasing distal edema. She was affected by chronic cardiac failure with Ejection Fraction (EF) between 40 and 45\% due to chronic cardiac ischemia with a previously NSTEMI treated conservatively in 2015, chronic hypertension, initial cognitive impairment; in 2016 she had acute pulmonary embolism caused by proximal femoral vein thrombosis, in 2017 she underwent abdominal aortic endoprothesis replacement for aortic abdominal aneurism. She was in therapy with Direct Oral Anticoagulant (DOAC), diuretic, ACEinhibitors and beta blockers. At the arrival in ED: arterial pressure was $85 / 65 \mathrm{mmHg}$, EKG showed atrial fibrillation with 110 beats per min with diffuse inferolateral nonspecific alterations; temperature was $38.6^{\circ}$, $\mathrm{sO} 2$ in oxygen mask at $\mathrm{FiO} 2$ 50\% was 90\%; she was dyspneic with a respiratory rate of 32 breaths per min, the skin was pale and wet; vesicular murmur was absent at both lung bases and there were crackles at the both middle lung fields; finally, there were moderate bilateral distal edema.

The anteroposterior Chest X-Ray (CXR) showed bilateral pleural effusion at the inferior side and blood tests (Table 1) revealed WBC 19500/mmc, CRP $70 \mathrm{mg} / \mathrm{dL}$ (n.v. $<5 \mathrm{mg} / \mathrm{dL}$ ), normal renal function, CPK $800 \mathrm{IU} / \mathrm{L}$ (n.v. $<135 \mathrm{U} / \mathrm{L}$ ), sodium 145 $\mathrm{mEq} / \mathrm{L}$, potassium $3.8 \mathrm{mEq} / \mathrm{L}$ and troponin $8 \mathrm{ng} \mu / \mathrm{L} / \mathrm{L}$ (n.v. $<$ $0.012 \mathrm{ng} / \mathrm{L}$ ). Blood gas analysis in ambient air showed hypoxemia at $58 \mathrm{mmHg}$, pH 7.35, pCO2 $35 \mathrm{mmHg}, \mathrm{HCO} 322 \mathrm{mEq} / \mathrm{L}$ and lactate was $3.2 \mathrm{mmol} / \mathrm{L}$.

We stopped ACE-inhibitors and beta blockers and started giving her oxygen at 50\% FiO2 with facial mask, $1000 \mathrm{~mL}$ crystalloids in about 60 minutes and paracetamol $500 \mathrm{mg}$, without any change in blood pressure and heart rate. In the mean time we performed a lung ultrasound (LUS) showing bilateral pleural effusion of three spaces and B lines in both medial fields with subpleural consolidation with dynamic air bronchogram compatible with pneumonia on the right side (not visible in CXR because of concomitant pleural effusion). We performed a cardiac ultrasound, that showed a dilated left ventricle with an inspective moderate low $\mathrm{EF}$; inferior vena cava of $22 \mathrm{~mm}$, with distension of hepatic veins and collapse index of less than $30 \%$ with an estimated central venous pressure (CVP) of about $18 \mathrm{mmHg}$. To quantify the SV, we used the LVOT-VTI (Figure 1). In this way, according to echocardiography guidelines (1) first we calculated aortic LVOT diameter as $19 \mathrm{~mm}$, then cardiac output (CO) as $2.8 \mathrm{~L} / \mathrm{min}(4-6 \mathrm{~L} / \mathrm{min})$ (using the formula $\mathrm{CO}=$ heart rate $\mathrm{x}$ (LVOT-VTI $\mathrm{x}$ aortic area), as showed in Figure 1, and then we derived the peripheral vascular resistance (PVR) as about 2100 dynes.sec/ $\mathrm{cm}^{5}$ (normal range 8001400 dynes.sec $\left./ \mathrm{cm}^{5}\right)$, using the formula PVR $=[(\mathrm{MAP}-$ $\mathrm{CVP}) / \mathrm{CO}$ ] 80 , (where MAP $=$ mean arterial pressure and $\mathrm{CVP}=$ central venous pressure). The SOFA score, calculated because of the pneumonia seen with LUS, was 4 point, resulting in a diagnosis of sepsis. Unfortunately, we didn't perform ultrasound evaluation before fluid expansion and so we could not compare the data, but because we had no modifications in arterial pressure, and relying on all echo data previously showed, we could suppose that this 
patient was more full than empty, because of acute heart failure due to septic myocardial depression for pneumonia and quite high ventricular rate atrial fibrillation: for this reason we need to keep monitoring $\mathrm{CO}$. To ameliorate lung failure we started using CPAP with helmet with a PEEP of $10 \mathrm{cmH} 20$ and $\mathrm{FiO} 250 \%$; to improve cardiac function, relying on the reduced LVOT-VTI and clinical evaluation, we stopped infusions and gave diuretic (furosemide 40 mg ev twice a day) because we have supposed that she was in the flat part of the Starling curve. We administered antibiotic therapy for community-acquired pneumonia as well. Arterial pressure stabilized at 95/55 $\mathrm{mmHg}$, and we did not start norepinephrine considering the high value of PVR we found and fearing a further increase of it with probably decrease of $\mathrm{CO}$.

During the first 24 hours she appeared to get slightly better: arterial pressure progressively rose up and stabilized to $95 / 60$ $\mathrm{mmHg}$, urine output was $1800 \mathrm{~mL} /$ day, heart rate was reduced at 90 beats per min (permanently in atrial fibrillation) and oxygen saturation was improved to $95 \%$ in CPAP helmet. We repeated cardiac ultrasound and found that LVOT-VTI had improved from 9 to $12 \mathrm{~cm}, \mathrm{CVP}$ was estimated at about $15 \mathrm{mmHg}(18 \mathrm{~mm}$ and collapse index at $<30 \%$ ) CO was estimated as $3.1 \mathrm{~L} / \mathrm{min}$ and PVR as 1500 dynes.sec/ $/ \mathrm{cm}^{5}$, so we kept going on with the same therapy.

Between day 2 and 5 we stopped CPAP, rhythm returned spontaneously sinus at 75 beats per min with minimal $\mathrm{T}$ alterations and troponin level decresead; Arterial pressure was stable at 105/50 $\mathrm{mmhm}$, LVOT-VTI rose up to $15 \mathrm{~cm}$, with an estimated CO of 3.3 $\mathrm{L} / \mathrm{min}$ inferior vena cava of $15 \mathrm{~mm}$, without distension of hepatic veins, collapse index of $50 \%$ with an estimated CVP of about 10 $\mathrm{mmHg}$ and PVR decreased to 1300 dynes.sec/ $\mathrm{cm}^{-5}$. After a constant improvement during the following week, we discharged the patients at day 9 .
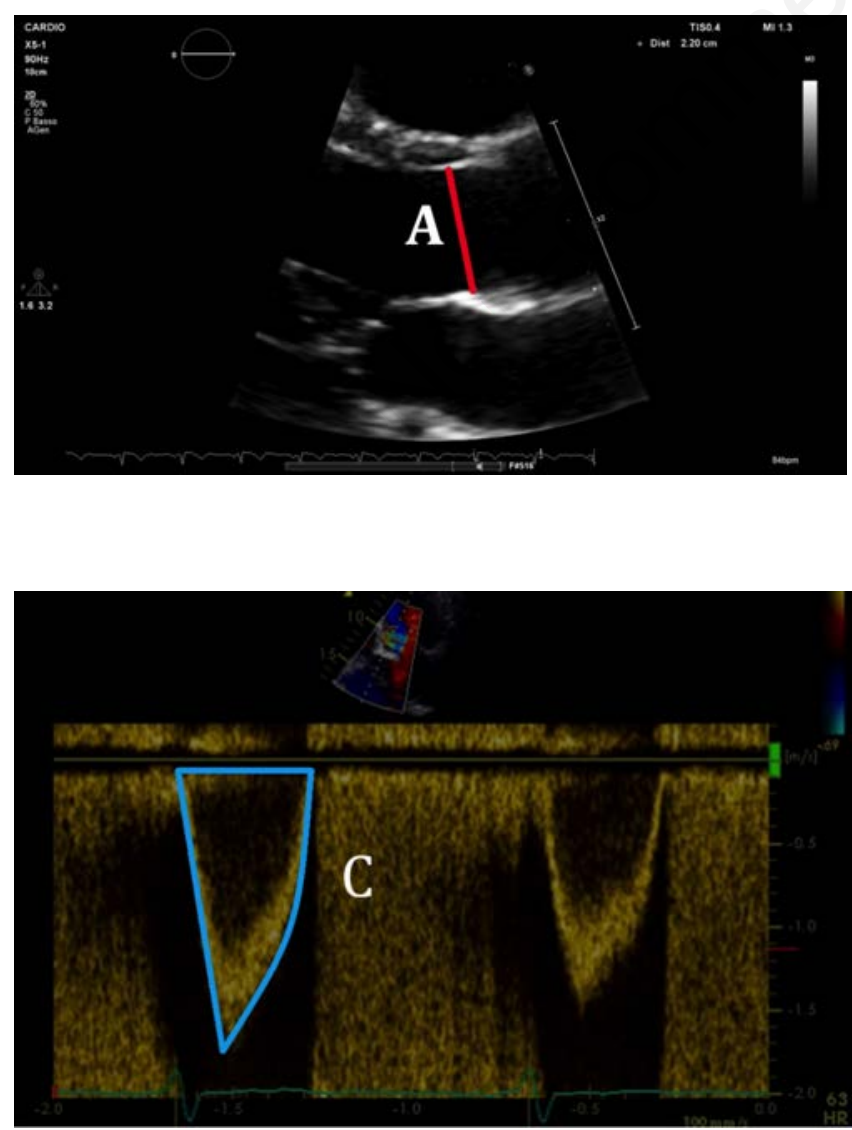

In Figure 2 we show the patient's hemodynamic trend (from A to $\mathrm{C}$ ) we have presumed during the hospitalization using $\mathrm{CO}$ assessment from LVOT-VTI and CVP estimated from inferior vena cava collapsibility.

\section{Discussion}

Fluid administration is a cornerstone of sepsis and septic shock therapy: anyway, it should be considered that an excessive amount of fluid, especially in a condition of capillary leak like sepsis, can lead to water extravasation, interstitial oedema and then organ dysfunction, with potential harmful consequences. ${ }^{1}$ Quite often, physiopathological mechanisms in peri-shock and shock states are more complex than they appear and they can involve more than one of the three major hemodynamic mainstays: volemia, myocar-

Table 1. Blood test.

\begin{tabular}{lcccc} 
& Day 1 & Day 2 & Day 3-5 & Day 9 \\
Hb g/dL & 11.2 & 10.6 & 10.2 & 10 \\
WBC cell/uL & 19.500 & 18.500 & 14.000 & 9.000 \\
\hline CRP $(<5 \mathrm{mg} / \mathrm{dL})$ & 70 & & 45 & 20 \\
Creatinine $(\mathrm{mg} / \mathrm{dL})$ & 1.1 & 1.4 & 1 & 0.9 \\
\hline CPK $(<180 \mathrm{UI})$ & 800 & 450 & & 225 \\
ALT/AST $(\mathrm{IU} / \mathrm{L})$ & $55 / 75$ & $60 / 80$ & $35 / 40$ & $28 / 30$ \\
\hline Troponin $(<0.012 \mathrm{ng} / \mathrm{mL})$ & 8 & 8 & 3 & 0.5 \\
\hline
\end{tabular}

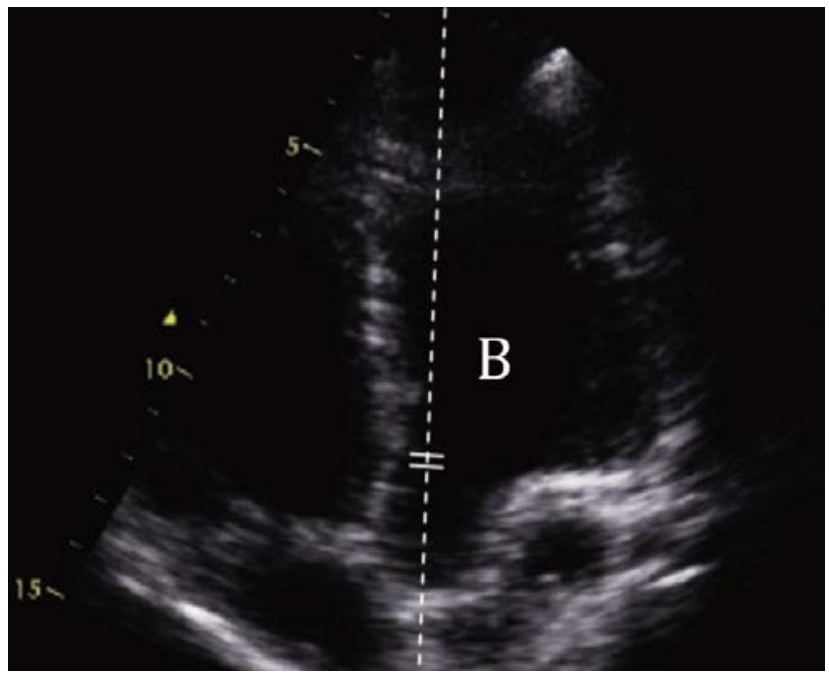

Figure 1. How to calculate cardiac output using aortic VTI. To calculate the stroke volume and then the cardiac output, the first step is measuring the diameter of the LVOT (A): this can be performed in the parasternal long axis view, proximal to the aortic valve, in mid-sistole. Once obtained the diameter, we can calculate the cross-sectional area (CSA). VTI is rec-orded with pulsed Doppler from an apical fivechamber view (B). Then the pulsed Doppler sample volume has to be positioned proximal to the aortic valve and in line with the blood flow through the LVOT. When the sample volume is correctly positioned, the recording shows a velocity curve with a well-defined peak; the VTI is then measured ( $\mathrm{C}$ in blu line). Assuming the stroke volume as a cylinder, it can be calculated according to the formula $S V=C S A$ (base) $x$ VTI (height). 
dial function and vascular tone. In our case we had to face the need for a very conservative fluid resuscitation, considering that, beyond the comments about water extravasation above mentioned ("volemia"), the patient had an acute-on-chronic heart failure and probably a septic myocardial depression ("contractility"): in other words, do we have to administer crystalloids or not in a patient that is on the flat part of the Frank-Starling curve? In order to answer to this question properly, it is mandatory to assess accurately the exact degree of each of these components.

We need also to remember that $\mathrm{CO}$ is the main determinant of oxygen delivery and the result of ventricular Stroke Volume (SV) and Heart Rate (HR). Thus, the assessment and optimization of CO are recommended in critical care ill patients with altered tissue perfusion. ${ }^{2}$

Arterial pressure, although is the single most common parameter used worldwide in ED to guide cardiovascular assessment, is not the only answer to guide fluid resuscitation in difficult scenarios, because of the complex physiology behind its value involving $\mathrm{CO}$, vascular tone and elastance of the arteries; ${ }^{3}$ we also know it could be misleading in acute settings: for example, a normal arterial pressure is possible in a patient who has high vascular tone and low $\mathrm{CO}$ or, at the opposite side, very low vascular tone and high $\mathrm{CO}$, as it happens in the early phase of septic shock. ${ }^{4}$

Clinical echocardiography in ED settings has a well definite role. ${ }^{5,6}$ What is interesting is that in emergency scenarios it is not necessary to reach the expert cardiology physician level, because it is generally enough to watch for general cardiac function, heart chambers dimension and valves integrity using basic view. However, we need also something that could give us a "numeric value" of the cardiac function, in an easy way. EF is the most common single echocardiographic parameter used worldwide, but it has several limitations: it could overestimate the actual cardiac function in case of mitral regurgitation and sometime could have problems in manual delineations of the endocardium with boundary continuity in critical patients, ${ }^{7}$ therefore the correct interpreta- tion of EF requires adequate experience. LVOT-VTI needs less ultrasound skill, is easily reproducible and could quickly and noninvasively estimate $\mathrm{CO}$ and its variations after fluid challenge vasoactive therapy. ${ }^{2,8}$ It should be considered, anyway, that LVOTVTI measure has to be very accurate, since even a few millimeters of error in the measurement of the outflow tract of the left ventricle, having to raise the radius to the second power, can cause considerable errors: this suggest us that we could rely only on VTI variations. With $\mathrm{CO}$ data and the inferior vena cava collapsibility with echo as an indirect value of CVP, we could also obtain a rough general value of the systemic vascular resistances as mentioned above. Finally, in ED settings is not easily possible to estimate in which part of the CO/CVP relation the patient is, according to the Frank-Starling law: in this patient we relied on LVOT-VTI value together with clinical and others ultrasound parameters, to better understand and follow patient's hemodynamic profile and each day response to therapy. If we had based our hemodynamic evaluation only on clinical exam and clinical parameters, we could have taken a different way to treat this patient, probably using more fluids and therefore worsening the condition of heart failure: this is a critical issue especially in septic patients, considering that up to $60 \%$ of them suffer from myocardial depression ${ }^{9}$ although its clinical meaning is still subject of debating. ${ }^{10}$

\section{Conclusions}

In selected cases in ED, in which there could be different causes of instability induced by a modification of two or more between vascular tone, cardiac function and volemia, we think that the use of LVOT-VTI as we showed in this case report, could be an useful tool for ED physician, partly thanks to its reproducibility and simple use, but mostly because it could guide the clinician to better understand the pathophysiology behind the single case and monitor adequately patients' response to therapy.

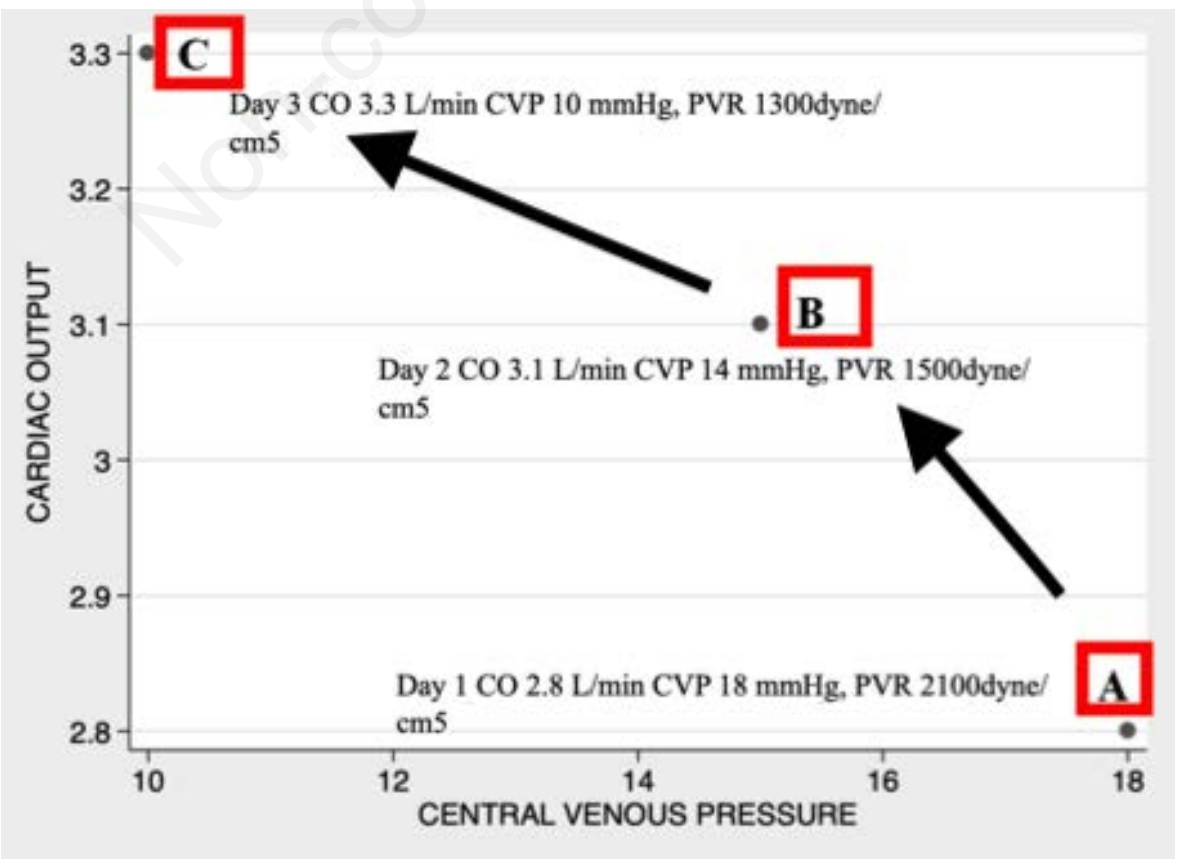

Figure 2. Patient's hemodynamic trend during the first three days derived from LVOT-VTI and clinical data. 


\section{References}

1. De Backer D, Cholley P, Slama M, Vieillard-Baron A, Vignon P. Hemodynamic monitoring us-ing echocardiography in the critically ill. Springer; 2011.

2. Malbrain M, Van Regenmortel N, Saugel B, et al. Principles of fluid management and steward-ship in septic shock: it is time to consider the four D's and the four phases of fluid therapy. Ann Intensive Care 2018;8:66 .

3. Magder S. The meaning of blood pressure. Crit Care 2018;22:257 .

4. Pinsky MR, Teboul JL, Vincent JL. Hemodynamic Monitoring. Springer; 2019.

5. Viellard-Baron A, Millington SJ, Sanfilippo F, Chew FM, et al. A decade of progress in critical care echocardiography: a nar.

rative review. Intensive Care Med 2019;45:770-88.

6. Pinsky MR, Payen D. Functional hemodynamic monitoring. Crit Care 2005;9:566-72.

7. Cikes M, Solomon SD. Beyond ejection fraction: an integrative approach for assessment of cardiac structure and function in heart failure. Eur Heart J 2015;37:1642-50.

8. Cholley B. Echocardiography in the intensive care unit: beyond "eyeballing". A plea for the broader use of the aortic velocity-time integral measurement. Intensive Care Med 2019;45:898-901

9. Vieillard-Baron A, Caille V, Charron C, Belliard G, et al. Actual incidence of global left ven-tricular hypokinesia in adult septic shock. Crit Care Med 2008;36:1701-06

10. McLean SA. Down but not out: myocardial depression in sepsis. Crit Care 2012;16:132. 